Paediatr Paedolog 2017 · 52:83

DOI 10.1007/s00608-017-0486-9

Online publiziert: 14. Juni 2017

(c) Springer-Verlag Wien 2017

CrossMark

Die Problematik der ernährungsabhängigen Erkrankungen („non-communicable diseases") ist seit Jahren bei internationalen Gesundheitsorganisationen (WHO; EU etc.) ein Topthema; Hintergrund ist, dass Herz-KreislaufErkrankungen in den Industrieländern Todesursache Nummer 1 sind und der ungesunde Lifestyle (Überernährung und mangelnde körperliche Aktivität) als verursachender Faktor identifiziert worden ist. Neueste Statistiken zeigen klar auf, dass in den meisten Ländern der Welt, aber v. a. in Mitteleuropa die Prävalenzen des Übergewichts, der Adipositas und insbesondere der extremen Adipositas stark im Steigen begriffen sind [1].

Die Folgen davon sind ein dramatischer Anstieg des Diabetes Typ II, der Herz-Kreislauf-Erkrankungen, Gelenkerkrankungen, einiger Krebsarten etc. Die wirtschaftlichen Folgen sind überaus besorgniserregend und drohen, die Gesundheitssysteme wesentlich $\mathrm{zu}$ überfordern [2]. Da die Therapie der höhergradigen Adipositas sowohl bei Erwachsenen als auch bei Kindern und Jugendlichen wenig erfolgreich ist und praktisch als gescheitert angesehen werden muss, hat die WHO Europa für die Mitgliedsländer klare Strategien zur Prävention und Beendigung der Übergewichtsproblematik entworfen und den Ländern die Umsetzung dieser aufgetragen (Report of the Commission on Ending Childhood Obesity: Implementation plan, WHO Executive Board 140th session, 13th Jan 2017).

Die Kernpunkte dieses Papiers können folgendermaßen zusammengefasst werden: „Take ownership, provide leadership and engage political commitment to tackle childhood obesity over the long

\title{
Kurt Widhalm
}

Wien, Österreich

\section{Das Wesentlichste ist das Commitment!}

term“, das ist die „main message“, die ernst genommen werden sollte.

An Einzelmaßnahmen sind folgende Punkte genannt:

- Sicherstellung von Daten über BodyMass-Index, Größe und Gewicht bei allen Kindern und Jugendlichen

- Programme zur Erhöhung der körperlichen Aktivität

- Entwicklung und Implementierung von evidenzbasierten Public-education-Programmen über gesunde Ernährung und deren Benefits, und eine Erhöhung der physischen Aktivität

- Einführung von wirksamen Steuern für zuckerhaltige Getränke

- Implementierung von Monitorsystemen, die die Wirkung der Maßnahmen überprüfen und politische Ziele festlegen sollen

Im Detail ist eine Vielzahl von Maßnahmen genannt, die geeignet sein können, die Ziele zu erreichen; das Wesentlichste ist jedoch das Commitment!

In dieser Hinsicht besteht in Österreich erheblicher Nachholbedarf: Es gibt weder von politischer Seite, noch von Sozialversicherungen, Ärztekammer, Fachgesellschaften etc. ein klares Commitment mit einem detaillierten Durchführungsplan. Hier ist auch die Pädiatrie aufgerufen, sich konkret zu engagieren, ein Commitment zu statuieren, nachweisbar wirksame Programme zu etablieren und in die Wege zu leiten. Dazu müssen die Methoden nicht neu erfunden werden, es gibt genügend Literatur, die wirksame Maßnahmen aufzeigt.

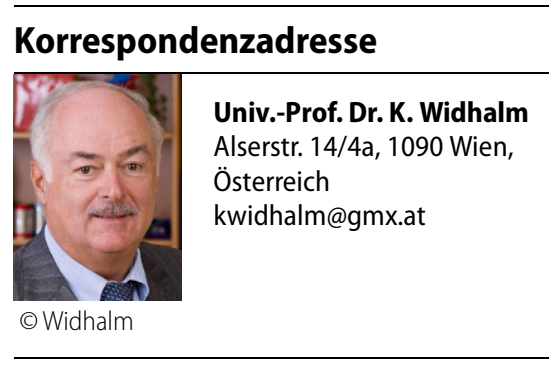

Univ.-Prof. Dr. Kurt Widhalm Präsident des Österreichischen Akademischen Instituts für Ernährungsmedizin

Interessenkonflikt. K. Widhalm gibt an, dass kein Interessenkonflikt besteht.

\section{Literatur}

1. NCD Risk Factor Collaboration (NCD-RisC) (2016) Trends in adult body-mass index in 200 countries from 1975 to 2014: a pooled analysis of 1698 population-based measurement studies with 19.2 million participants. Lancet 387:1377-1396

2. Yates Netal (2013) The economic burden of obesity in Germany: results from the population-based KORA studies. Obes Facts 9(6):397-409. doi:10. $1159 / 000452248$ 